\title{
Perspectives of Physicians and Nurses Regarding End-of-Life Care at King Abdullah Medical City in Makkah: A Cross-Sectional Study
}

\section{Naved Yousuf Hasan ${ }^{1 *}$, Asiah Rugaan ${ }^{2}$, Masood Iqbal ${ }^{3}$ and Sanaullah Bhatti $^{4}$}

${ }^{1}$ American Board of Internal Medicine, Pulmonary and Critical Care Medicine, Consultant, Department of Intensive Care, King Abdullah Medical City, Makkah, Saudi Arabia

${ }^{2}$ Saudi Board of Internal Medicine and Adult Intensive Care, Director and Consultant, Department of Intensive Care, King Abdullah Medical City, Makkah, Saudi Arabia ${ }^{3}$ Diploma in Anesthesiology, Assistant Consultant, Department of Intensive Care, King Abdullah Medical City, Makkah, Saudi Arabia

${ }^{4}$ MRCS Surgery, Assistant Consultant, Department of Intensive Care, King Abdullah Medical City, Makkah, Saudi Arabia

*Corresponding Author: Naved Yousuf Hasan, American Board of Internal Medicine, Pulmonary and Critical Care Medicine, Consultant, Department of Intensive Care, King Abdullah Medical City, Makkah, Saudi Arabia.
Received: July 19, 2021

Published: August 30, 2021

(C) All rights are reserved by Naved Yousuf

Hasan., et al.

\section{Abstract}

End of life care decision is challenging. We assessed the perspectives of physicians and nurses in a tertiary care hospital by testing 19 statements. A total of 112 physicians and 188 nurses completed the survey. Both physicians and nurses agreed to 15 statements, disagreed to 2 , and regarding statements 4 and 7 , nurses were having a different opinion than physicians. In particular, nurses were more likely to agree while physicians disagree. According to our results, one reason for transferring sick patients to ICU is the fear of legal implications. Physicians and nurses both agreed that patients and families were encouraged to make timely and appropriate decisions about DNR and their religious beliefs greatly influence their views regarding EOLC decisions. Furthermore, physicians do talk about health care directives with patients and family members regularly. Nurses agreed that the patients with DNR status should always be treated in the intensive care unit if indicated.

On the other hand, physicians do not want to treat DNR patients in ICU (P = 0.003). Similarly, nurses agreed that we routinely placed a religious assessment of the patients in patient charts and nurses/physicians know about it while physicians do not agree with that $(\mathrm{P}=0.000)$. It is a single-center study, hence the generalization of its results is limited. We did not calculate the actual practices of providing EOLC in our hospital. The possible confounding factors, such as age, gender, and ethnicity, have not been addressed in our survey.

Keywords: Do not Resuscitate; Dying Patients; End of Life; Perspective of Physicians and Nurses 


\section{Background and Rationale}

Decisions regarding providing end-of-life care (EOLC), including the withholding and withdrawing of life support, is a significant challenge that faces patients, their relatives, and intensive care physicians $[1,2]$. The complex interaction between the ethical, social, cultural, moral, and religious values besides the patients' prognosis, overall performance, and quality of life before hospital admission plays a major role in these hard decisions $[3,4]$.

The ability of an intensive care physician to provide competent end-of-life care (EOLC) is an important skill to reach the optimal EOLC, which requires a perspective provided by multiple members of the medical team [5]. Of course, leadership, deliberation, and explicit collaboration are all needed for the optimal implantation of EOLC [6-9].

Worldwide, the demand for critical care services is increasing, which is, according to researchers, attributed to the increase of the population age, longer survival of previously thought incurable diseases, and the mandatory post-operative intensive care admission [10]. Also, health organizations and hospital administrations are pressuring physicians to act more cost-effectively.

We have found that physicians and nurses have different views regarding EOLC, even though both are heading toward the same goal. Most physicians postpone discussing DNR until the patient is showing a poor prognosis or when his condition worsens, while nurses approach this issue early when we admit the patient, which is attributed to the fact that physicians are probably more optimistic regarding the prognosis than nurses [11].

Saudi Arabia is a country that abides by very strict religious and cultural values. Thus, many Saudi hospitals have adopted a DNR policy that follows the religious point of view regarding (fatwa) DNR, which was established in 1988 and it showed that we do not require family opinion for EOLC decision, it mainly depends upon three knowledgeable and trustworthy physicians involved in patient care $[12,13]$.

Unfortunately, we lack adequate researches regarding end-oflife care in the Middle East, and international researches are usually not applicable because of the beforehand mentioned different cultural and religious background [14]. In general, we found that in Saudi Arabia, the concern of physicians was more about religion and litigation than the cost of care [15].

\section{Aim and Objectives}

- $\quad$ Primary: To measure the perception of physicians and nurses regarding EOLC at King Abdullah Medical City (KAMC) in the holy capital.

- Secondary: To compare the views of consultants, specialists, and resident physicians regarding EOLC and whether DNR policies are being applied in KAMC. To compare the views of physicians with nurses regarding EOLC.

\section{Methodology}

Study design

A cross-sectional observational study.

Study population

Inclusion criteria

Physicians (Consultants, specialists, and residents) and nurses working at the clinical and critical care departments at KAMC during June 2016.

\section{Exclusion criteria}

Physicians and nurses who don't agree to take part.

Study procedure

- $\quad$ The survey used is a modified preexisting survey on EOLC in ICU adapted from Emir Festic., et al. research in 2012 with additional statements.

- A list of physicians and nurses who met the inclusion criteria was obtained from the department of human resources/staff affairs.

- We delivered a self-administered questionnaire randomly to the targeted sample size at their departments. They filled the questionnaire with the following information:

- Demographic information: Specialty, Position of the participants, Years of experience.

- Views regarding specific statements (19 statements and 4 yes/no questions) on the perspective, attitude, and practice of DNR policies at KAMC. The participants recorded their views on a Likert-scale form, containing all statements (Attachment I). 
Outcome assessment

Primary outcome: the mean of the average mean score of Likert scale of different statements.

Data collection and management

Our study was a single-center cross-sectional study from June $1^{\text {st }}$ to June $30^{\text {th }}, 2016$. After getting KAMC IRB approval, we started our data collection on hard copies. Data was collected from the physicians and nurses after they filled the questionnaire, not showing any nominative information. We entered the data into the SPSS system. The names of the participants were kept anonymous. Two persons from the research team handled data entry and data. A KAMC statistician analyzed the data to get the results. We will publish the results in local and international journals.

\section{Sample size determination}

We calculated the Sample size for a descriptive study of a continuous variable using the following equation:

Sample size $=\mathrm{N}=4 \mathrm{Z} \alpha 2 \mathrm{~S} 2 / \mathrm{W} 2=61$.

Considering $\mathrm{Cl}=95 \%, \mathrm{~W}=2$ and $\mathrm{S}=1$.

Compare the mean of continuous measurement in two samples, using a z-statistic to approximate the t-statistic, the sample size was calculated as follows:

The standard normal deviate for $\alpha=\mathrm{Z}_{\alpha}=1.960$.

The standard normal deviate for $\beta=\mathrm{Z}_{\beta}=0.842$.

$A=\left(1 / q_{1}+1 / q_{0}\right)=6.250$.

$\mathrm{B}=\left(\mathrm{Z}_{\alpha}+\mathrm{Z}_{\beta}\right)^{2}=7.849$.

Standardized Effect Size $=(E / S)=0.500$.

Total group size $=\mathrm{N}=\mathrm{AB} /(\mathrm{E} / \mathrm{S})^{2}=196.22$.

Considering $\mathrm{q} 1=0.2$ and $\mathrm{S}=1$.

$\mathrm{N}_{1}: 39$ (ICU).

$\mathrm{N}_{0}: 157$ (Non ICU).

Total: 196.

\section{Statistical analysis plan}

We analyzed the obtained data on SPSS V22.8. Numeric data were presented as mean $\pm \mathrm{SD}$, or as median and range according to the type of distribution of each variable. For categorical variables, percentages were used. Comparison between groups was made by Wilcoxon Rank Sum test or Mann-Whitney U test according to data distribution. A Chi-squared test was used for categorical values.

A regression model was constructed with the mean of questions as the dependent variable. The model was linear or ordinal according to the distribution of the outcome variable and the fulfillment of the outcome assumption. Different demographic factors were entered individually into the model, and significant ones $(\mathrm{P}<0.1)$ were entered into a multifactorial model. Ordinal regression was performed on Stata V.13.

\section{Ethical part and confidentiality}

Ethical approval was sought from KAMC Institutional Review Board (IRB). The subjects were included in the study after taking verbal consent.

\section{Results}

Response rate

\begin{tabular}{|l|c|c|}
\hline \multicolumn{3}{|c|}{ Response Rate } \\
\hline Physicians & $128 / 143$ & $89.51 \%$ \\
\hline Nurses & $188 / 250$ & $75.2 \%$ \\
\hline Total & $316 / 393$ & $80.40 \%$ \\
\hline
\end{tabular}

Table 1

\begin{tabular}{|l|c|c|}
\hline Demographics of physicians & $\#$ & \% \\
\hline Reported positions & 110 & $98.21 \%$ \\
\hline Consultant & 21 & $18.75 \%$ \\
\hline Specialist & 69 & $61.60 \%$ \\
\hline Residents & 20 & $17.86 \%$ \\
\hline Not reported & 2 & $1.79 \%$ \\
\hline Total & 112 & $100 \%$ \\
\hline Total reported specialty & 112 & $100 \%$ \\
\hline Critical care & 19 & $16.96 \%$ \\
\hline Oncology & 5 & $4.47 \&$ \\
\hline Non-critical care non oncology & 88 & 78.57 \\
\hline Not reported & 0 & $0 \%$ \\
\hline Total & 112 & $100 \%$ \\
\hline
\end{tabular}

Table 2 


\begin{tabular}{|l|c|c|}
\hline Demographics of nurses & $\#$ & \% \\
\hline The reported positions & 181 & $96.27 \%$ \\
\hline Critical & 64 & $34.04 \%$ \\
\hline Oncology & 38 & $20.21 \%$ \\
\hline Non-critical & 79 & $42.02 \%$ \\
\hline Not reported & 7 & $3.73 \%$ \\
\hline Total & 188 & $100 \%$ \\
\hline The reported level of education & 175 & $93.08 \%$ \\
\hline Diploma & 12 & $6.38 \%$ \\
\hline Bachelor & 158 & $84.05 \%$ \\
\hline Master & 5 & $2.66 \%$ \\
\hline Not reported & 13 & $6.91 \%$ \\
\hline Total & 188 & $100 \%$ \\
\hline
\end{tabular}

Table 3

\begin{tabular}{|c|c|c|c|c|c|}
\hline \multirow[b]{2}{*}{ Statement } & \multicolumn{5}{|c|}{ Responses } \\
\hline & $\begin{array}{l}\text { Strongly } \\
\text { disagree }\end{array}$ & $\begin{array}{c}\text { Dis- } \\
\text { agree }\end{array}$ & $\begin{array}{c}\text { Neu- } \\
\text { tral }\end{array}$ & Agree & $\begin{array}{l}\text { Strong- } \\
\text { ly agree }\end{array}$ \\
\hline \multicolumn{6}{|l|}{$\begin{array}{l}\text { Fear of legal } \\
\text { implications } \\
\text { often results } \\
\text { in transferring } \\
\text { dying patients } \\
\text { to ICU }\end{array}$} \\
\hline Physicians & 9 & 15 & 29 & 54 & 19 \\
\hline Consultants & 1 & 4 & 6 & 13 & 5 \\
\hline Specialists & 7 & 9 & 15 & 33 & 13 \\
\hline Residents & 1 & 2 & 8 & 8 & 1 \\
\hline P-value & 0.510 & & & & \\
\hline Nurse & 3 & 16 & 63 & 71 & 19 \\
\hline Critical & 1 & 2 & 18 & 30 & 9 \\
\hline Oncology & 2 & 6 & 13 & 10 & 5 \\
\hline $\begin{array}{l}\text { Non-critical Non- } \\
\text { Oncology }\end{array}$ & 0 & 8 & 32 & 31 & 5 \\
\hline P-value & 0.028 & & & & \\
\hline 5 years or less & 0 & 5 & 28 & 18 & 6 \\
\hline 6-15 years & 3 & 11 & 35 & 54 & 12 \\
\hline $\begin{array}{l}\text { More than } 15 \\
\text { years }\end{array}$ & 0 & 1 & 1 & 0 & 0 \\
\hline P-value & 0.130 & & & & \\
\hline $\begin{array}{l}\text { Total (Physicians } \\
\text { and Nurses) }\end{array}$ & 12 & 31 & 92 & 125 & 38 \\
\hline
\end{tabular}

\begin{tabular}{|c|c|c|c|c|c|}
\hline P-value & 0.774 & & & & \\
\hline \multicolumn{6}{|l|}{$\begin{array}{l}\text { Patients and } \\
\text { families are } \\
\text { encouraged to } \\
\text { make timely and } \\
\text { appropriate deci- } \\
\text { sions about DNR } \\
\text { orders }\end{array}$} \\
\hline Physicians & 10 & 15 & 27 & 56 & 20 \\
\hline Consultants & 2 & 4 & 9 & 9 & 6 \\
\hline Specialists & 6 & 8 & 9 & 43 & 12 \\
\hline Residents & 2 & 3 & 9 & 4 & 2 \\
\hline P-value & 0.060 & & & & \\
\hline Nurse & 5 & 12 & 22 & 96 & 44 \\
\hline Critical & 3 & 7 & 7 & 32 & 13 \\
\hline Oncology & 1 & 1 & 3 & 21 & 12 \\
\hline $\begin{array}{l}\text { Non-critical Non- } \\
\text { Oncology }\end{array}$ & 0 & 0 & 13 & 40 & 25 \\
\hline P-value & 0.183 & & & & \\
\hline 5 years or less & 1 & 2 & 6 & 34 & 16 \\
\hline 6-15 years & 4 & 10 & 18 & 61 & 25 \\
\hline $\begin{array}{l}\text { More than } 15 \\
\text { years }\end{array}$ & 0 & 0 & 0 & 1 & 1 \\
\hline P-value & 0.147 & & & & \\
\hline $\begin{array}{l}\text { Total (Physicians } \\
\text { and Nurses) }\end{array}$ & 15 & 27 & 49 & 152 & 64 \\
\hline P-value & 0.001 & & & & \\
\hline \multicolumn{6}{|l|}{$\begin{array}{l}\text { Physicians talk } \\
\text { about health care } \\
\text { directives with } \\
\text { patient and fam- } \\
\text { ily members }\end{array}$} \\
\hline Physicians & 2 & 4 & 18 & 73 & 28 \\
\hline Consultants & 0 & 0 & 4 & 17 & 6 \\
\hline Specialists & 2 & 2 & 9 & 45 & 20 \\
\hline Residents & 0 & 2 & 5 & 11 & 2 \\
\hline P-value & 0.105 & & & & \\
\hline Nurse & 5 & 2 & 29 & 88 & 54 \\
\hline Critical & 3 & 1 & 10 & 33 & 15 \\
\hline Oncology & 2 & 1 & 6 & 15 & 14 \\
\hline $\begin{array}{l}\text { Non-critical Non- } \\
\text { Oncology }\end{array}$ & 0 & 0 & 13 & 40 & 25 \\
\hline P-value & 0.432 & & & & \\
\hline
\end{tabular}

Citation: Naved Yousuf Hasan., et al. "Perspectives of Physicians and Nurses Regarding End-of-Life Care at King Abdullah Medical City in Makkah: A Cross-Sectional Study". Acta Scientific Medical Sciences 5.9 (2021): 156-166. 


\begin{tabular}{|c|c|c|c|c|c|}
\hline 5 years or less & 1 & 1 & 14 & 24 & 19 \\
\hline $6-15$ years & 4 & 1 & 16 & 63 & 33 \\
\hline More 15 years & 0 & 0 & 0 & 2 & 0 \\
\hline P-value & 0.926 & & & & \\
\hline $\begin{array}{l}\text { Total (Physicians } \\
\text { and Nurses) }\end{array}$ & 7 & 6 & 47 & 161 & 82 \\
\hline P-value & 0.355 & & & & \\
\hline $\begin{array}{l}\text { The patient with } \\
\text { DNR should al- } \\
\text { ways be treated } \\
\text { in intensive care } \\
\text { if indicated }\end{array}$ & & & & & \\
\hline Physicians & 21 & 44 & 24 & 34 & 5 \\
\hline Consultants & 3 & 13 & 3 & 7 & 4 \\
\hline Specialists & 17 & 24 & 15 & 21 & 1 \\
\hline Residents & 1 & 7 & 6 & 6 & 0 \\
\hline P-value & 0.374 & & & & \\
\hline Nurse & 11 & 49 & 53 & 50 & 14 \\
\hline Critical & 1 & 21 & 12 & 26 & 2 \\
\hline Oncology & 7 & 12 & 13 & 4 & 1 \\
\hline $\begin{array}{l}\text { Non-critical Non- } \\
\text { Oncology }\end{array}$ & 3 & 16 & 28 & 20 & 11 \\
\hline P-value & 0.001 & & & & \\
\hline 5 years or less & 4 & 8 & 21 & 18 & 6 \\
\hline $6-15$ years & 6 & 40 & 31 & 33 & 8 \\
\hline $\begin{array}{l}\text { More than } 15 \\
\text { years }\end{array}$ & 0 & 1 & 0 & 1 & 0 \\
\hline P-value & 0.234 & & & & \\
\hline $\begin{array}{l}\text { Total (Physicians } \\
\text { and Nurses) }\end{array}$ & 32 & 93 & 77 & 84 & 19 \\
\hline P-value & 0.003 & & & & \\
\hline \multicolumn{6}{|l|}{$\begin{array}{l}\text { My religious } \\
\text { beliefs greatly } \\
\text { influence my } \\
\text { view of DNR }\end{array}$} \\
\hline Physicians & 7 & 16 & 36 & 43 & 23 \\
\hline Consultants & 1 & 8 & 6 & 6 & 8 \\
\hline Specialists & 5 & 5 & 21 & 31 & 15 \\
\hline Residents & 1 & 3 & 9 & 6 & 0 \\
\hline P-value & 0.088 & & & & \\
\hline Nurse & 5 & 18 & 57 & 72 & 25 \\
\hline Critical & 0 & 8 & 20 & 27 & 6 \\
\hline Oncology & 3 & 3 & 10 & 16 & 5 \\
\hline
\end{tabular}

\begin{tabular}{|c|c|c|c|c|c|}
\hline $\begin{array}{l}\text { Non-critical Non- } \\
\text { Oncology }\end{array}$ & 2 & 7 & 27 & 29 & 14 \\
\hline P-value & 0.862 & & & & \\
\hline 5 years or less & 3 & 4 & 17 & 25 & 9 \\
\hline 6-15 years & 2 & 15 & 37 & 47 & 16 \\
\hline More 15 years & 0 & 0 & 1 & 1 & 0 \\
\hline P-value & 0.840 & & & & \\
\hline $\begin{array}{l}\text { Total (Physicians } \\
\text { and Nurses) }\end{array}$ & 12 & 34 & 93 & 115 & 48 \\
\hline P-value & 0.706 & & & & \\
\hline \multicolumn{6}{|l|}{$\begin{array}{l}\text { My cultural back- } \\
\text { ground makes it } \\
\text { difficult for me } \\
\text { to deal with the } \\
\text { DNR issue }\end{array}$} \\
\hline Physicians & 14 & 45 & 24 & 34 & 9 \\
\hline Consultants & 3 & 13 & 7 & 3 & 3 \\
\hline Specialists & 9 & 27 & 11 & 25 & 6 \\
\hline Residents & 2 & 5 & 6 & 6 & 0 \\
\hline P-value & 0.591 & & & & \\
\hline Nurse & 12 & 53 & 60 & 36 & 16 \\
\hline Critical & 1 & 20 & 22 & 13 & 6 \\
\hline Oncology & 3 & 12 & 13 & 7 & 2 \\
\hline $\begin{array}{l}\text { Non-critical Non- } \\
\text { Oncology }\end{array}$ & 8 & 21 & 25 & 16 & 8 \\
\hline P-value & 0.616 & & & & \\
\hline 5 years or less & 4 & 10 & 23 & 12 & 9 \\
\hline 6-15 years & 10 & 43 & 33 & 24 & 7 \\
\hline More 15 years & 0 & 0 & 1 & 1 & 0 \\
\hline P-value & 0.035 & & & & \\
\hline $\begin{array}{l}\text { Total (Physicians } \\
\text { and Nurses) }\end{array}$ & 26 & 98 & 84 & 70 & 25 \\
\hline P-value & 0.455 & & & & \\
\hline \multicolumn{6}{|l|}{$\begin{array}{l}\text { A religious } \\
\text { assessment of } \\
\text { the patient is } \\
\text { routinely placed } \\
\text { in patients charts } \\
\text { and nurses/ } \\
\text { physicians know } \\
\text { about it }\end{array}$} \\
\hline Physicians & 20 & 31 & 29 & 35 & 11 \\
\hline Consultants & 5 & 8 & 5 & 7 & 5 \\
\hline
\end{tabular}




\begin{tabular}{|c|c|c|c|c|c|}
\hline Specialists & 13 & 19 & 14 & 25 & 5 \\
\hline Residents & 2 & 4 & 10 & 3 & 1 \\
\hline P-value & 0.938 & & & & \\
\hline Nurse & 5 & 31 & 45 & 78 & 19 \\
\hline Critical & 2 & 15 & 7 & 30 & 7 \\
\hline Oncology & 1 & 6 & 10 & 19 & 2 \\
\hline $\begin{array}{l}\text { Non-critical Non- } \\
\text { Oncology }\end{array}$ & 2 & 10 & 28 & 29 & 10 \\
\hline P-value & 0.965 & & & & \\
\hline 5 years or less & 0 & 6 & 19 & 25 & 9 \\
\hline 6-15 years & 5 & 23 & 24 & 54 & 11 \\
\hline $\begin{array}{l}\text { More than } 15 \\
\text { years }\end{array}$ & 0 & 0 & 1 & 1 & 0 \\
\hline P-value & 0.438 & & & & \\
\hline $\begin{array}{l}\text { Total (Physicians } \\
\text { and Nurses) }\end{array}$ & 25 & 62 & 74 & 113 & 30 \\
\hline P-value & 0.000 & & & & \\
\hline \multicolumn{6}{|l|}{$\begin{array}{l}\text { Bereavement } \\
\text { and follow-up } \\
\text { services are } \\
\text { offered to family } \\
\text { members of pa- } \\
\text { tients who died } \\
\text { in the hospital }\end{array}$} \\
\hline Physicians & 12 & 20 & 35 & 50 & 8 \\
\hline Consultants & 4 & 7 & 5 & 10 & 4 \\
\hline Specialists & 8 & 11 & 20 & 33 & 3 \\
\hline Residents & 0 & 2 & 10 & 7 & 1 \\
\hline P-value & 0.914 & & & & \\
\hline Nurse & 3 & 15 & 57 & 89 & 14 \\
\hline Critical & 2 & 5 & 11 & 36 & 6 \\
\hline Oncology & 1 & 4 & 13 & 17 & 3 \\
\hline $\begin{array}{l}\text { Non-critical Non- } \\
\text { Oncology }\end{array}$ & 0 & 6 & 33 & 36 & 5 \\
\hline P-value & 0.176 & & & & \\
\hline 5 years or less & 0 & 4 & 23 & 28 & 5 \\
\hline 6-15 years & 3 & 12 & 33 & 60 & 8 \\
\hline $\begin{array}{l}\text { More than } 15 \\
\text { years }\end{array}$ & 0 & 0 & 1 & 0 & 1 \\
\hline P-value & 0.828 & & & & \\
\hline $\begin{array}{l}\text { Total (Physicians } \\
\text { and Nurses) }\end{array}$ & 15 & 35 & 92 & 139 & 22 \\
\hline
\end{tabular}

\begin{tabular}{|c|c|c|c|c|c|}
\hline P-value & 0.005 & & & & \\
\hline \multicolumn{6}{|l|}{$\begin{array}{l}\text { Services of a } \\
\text { social worker are } \\
\text { offered to dying } \\
\text { patients and } \\
\text { family members }\end{array}$} \\
\hline Physicians & 3 & 16 & 23 & 66 & 19 \\
\hline Consultants & 0 & 4 & 5 & 15 & 6 \\
\hline Specialists & 3 & 9 & 9 & 44 & 12 \\
\hline Residents & 0 & 3 & 9 & 7 & 1 \\
\hline P-value & 0.070 & & & & \\
\hline Nurse & 5 & 11 & 34 & 93 & 37 \\
\hline Critical & 2 & 9 & 9 & 34 & 8 \\
\hline Oncology & 1 & 0 & 5 & 20 & 12 \\
\hline $\begin{array}{l}\text { Non-critical Non- } \\
\text { Oncology }\end{array}$ & 2 & 2 & 20 & 39 & 17 \\
\hline P-value & 0.031 & & & & \\
\hline 5 years or less & 2 & 2 & 17 & 30 & 9 \\
\hline 6-15 years & 3 & 9 & 18 & 60 & 28 \\
\hline $\begin{array}{l}\text { More than } 15 \\
\text { years }\end{array}$ & 0 & 0 & 0 & 1 & 1 \\
\hline P-value & 0.187 & & & & \\
\hline $\begin{array}{l}\text { Total (Physicians } \\
\text { and Nurses) }\end{array}$ & 8 & 27 & 57 & 159 & 56 \\
\hline P-value & 0.122 & & & & \\
\hline \multicolumn{6}{|l|}{$\begin{array}{l}\text { Effective and } \\
\text { timely pain } \\
\text { management/ } \\
\text { comfort care } \\
\text { is provided to } \\
\text { patients who are } \\
\text { dying }\end{array}$} \\
\hline Physicians & 2 & 3 & 10 & 59 & 52 \\
\hline Consultants & 0 & 2 & 2 & 11 & 15 \\
\hline Specialists & 1 & 0 & 4 & 38 & 33 \\
\hline Residents & 1 & 1 & 4 & 10 & 4 \\
\hline P-value & 0.024 & & & & \\
\hline Nurse & 4 & 8 & 19 & 91 & 58 \\
\hline Critical & 4 & 2 & 1 & 37 & 18 \\
\hline Oncology & 0 & 1 & 1 & 21 & 15 \\
\hline $\begin{array}{l}\text { Non-critical Non- } \\
\text { Oncology }\end{array}$ & 0 & 5 & 17 & 33 & 25 \\
\hline P-value & 0.133 & & & & \\
\hline
\end{tabular}




\begin{tabular}{|c|c|c|c|c|c|}
\hline 5 years or less & 1 & 3 & 10 & 30 & 16 \\
\hline $6-15$ years & 3 & 5 & 9 & 60 & 41 \\
\hline $\begin{array}{l}\text { More than } 15 \\
\text { years }\end{array}$ & 0 & 0 & 0 & 1 & 1 \\
\hline P-value & 0.277 & & & & \\
\hline $\begin{array}{l}\text { Total (Physicians } \\
\text { and Nurses) }\end{array}$ & 6 & 11 & 29 & 150 & 110 \\
\hline P-value & 0.062 & & & & \\
\hline $\begin{array}{l}\text { I can safely raise } \\
\text { questions and } \\
\text { concerns about } \\
\text { end-of-life care } \\
\text { policies and } \\
\text { practices }\end{array}$ & & & & & \\
\hline Physicians & 0 & 9 & 34 & 63 & 20 \\
\hline Consultants & 0 & 1 & 7 & 14 & 8 \\
\hline Specialists & 0 & 3 & 24 & 37 & 12 \\
\hline Residents & 0 & 5 & 3 & 12 & 0 \\
\hline P-value & 0.074 & & & & \\
\hline Nurse & 2 & 11 & 42 & 100 & 23 \\
\hline Critical & 0 & 2 & 8 & 43 & 8 \\
\hline Oncology & 1 & 5 & 7 & 20 & 5 \\
\hline $\begin{array}{l}\text { Non-critical Non- } \\
\text { Oncology }\end{array}$ & 1 & 4 & 27 & 37 & 10 \\
\hline P-value & 0.054 & & & & \\
\hline 5 years or less & 0 & 3 & 18 & 32 & 7 \\
\hline $6-15$ years & 2 & 6 & 26 & 66 & 16 \\
\hline $\begin{array}{l}\text { More than } 15 \\
\text { years }\end{array}$ & 0 & 0 & 0 & 2 & 0 \\
\hline P-value & 0.716 & & & & \\
\hline $\begin{array}{l}\text { Total (Physicians } \\
\text { and Nurses) }\end{array}$ & 2 & 20 & 76 & 163 & 43 \\
\hline P-value & 0.939 & & & & \\
\hline \multicolumn{6}{|l|}{$\begin{array}{l}\text { Caring for dying } \\
\text { patients and } \\
\text { their families } \\
\text { is a rewarding } \\
\text { experience }\end{array}$} \\
\hline Physicians & 2 & 11 & 27 & 65 & 22 \\
\hline Consultants & 0 & 2 & 9 & 15 & 3 \\
\hline Specialists & 2 & 6 & 12 & 39 & 19 \\
\hline Residents & 0 & 3 & 6 & 11 & 0 \\
\hline
\end{tabular}

\begin{tabular}{|c|c|c|c|c|c|}
\hline P-value & 0.038 & & & & \\
\hline Nurse & 2 & 4 & 39 & 87 & 46 \\
\hline Critical & 2 & 3 & 8 & 35 & 14 \\
\hline Oncology & 0 & 0 & 13 & 17 & 7 \\
\hline $\begin{array}{l}\text { Non-critical Non- } \\
\text { Oncology }\end{array}$ & 0 & 1 & 18 & 35 & 25 \\
\hline P-value & 0.277 & & & & \\
\hline 5 years or less & 0 & 1 & 13 & 25 & 19 \\
\hline 6-15 years & 2 & 2 & 27 & 61 & 26 \\
\hline $\begin{array}{l}\text { More than } 15 \\
\text { years }\end{array}$ & 0 & 0 & 0 & 2 & 0 \\
\hline P-value & 0.506 & & & & \\
\hline $\begin{array}{l}\text { Total (Physicians } \\
\text { and Nurses) }\end{array}$ & 4 & 15 & 66 & 152 & 68 \\
\hline P-value & 0.046 & & & & \\
\hline \multicolumn{6}{|l|}{$\begin{array}{l}\text { The hospital } \\
\text { provides profes- } \\
\text { sional education } \\
\text { to physicians to } \\
\text { improve end- } \\
\text { of-life clinical } \\
\text { practices }\end{array}$} \\
\hline Physicians & 15 & 31 & 30 & 40 & 10 \\
\hline Consultants & 5 & 9 & 7 & 7 & 2 \\
\hline Specialists & 9 & 15 & 14 & 30 & 8 \\
\hline Residents & 1 & 7 & 9 & 3 & 0 \\
\hline P-value & 0.076 & & & & \\
\hline Nurse & 5 & 16 & 51 & 80 & 26 \\
\hline Critical & 2 & 6 & 13 & 33 & 8 \\
\hline Oncology & 1 & 3 & 12 & 19 & 3 \\
\hline $\begin{array}{l}\text { Non-critical Non- } \\
\text { Oncology }\end{array}$ & 2 & 7 & 26 & 28 & 15 \\
\hline P-value & 0.768 & & & & \\
\hline 5 years or less & 1 & 3 & 23 & 26 & 6 \\
\hline 6-15 years & 4 & 13 & 26 & 54 & 20 \\
\hline $\begin{array}{l}\text { More than } 15 \\
\text { years }\end{array}$ & 0 & 0 & 0 & 2 & 0 \\
\hline P-value & 0.540 & & & & \\
\hline $\begin{array}{l}\text { Total (Physicians } \\
\text { and Nurses) }\end{array}$ & 20 & 47 & 81 & 120 & 36 \\
\hline P-value & 0.000 & & & & \\
\hline
\end{tabular}




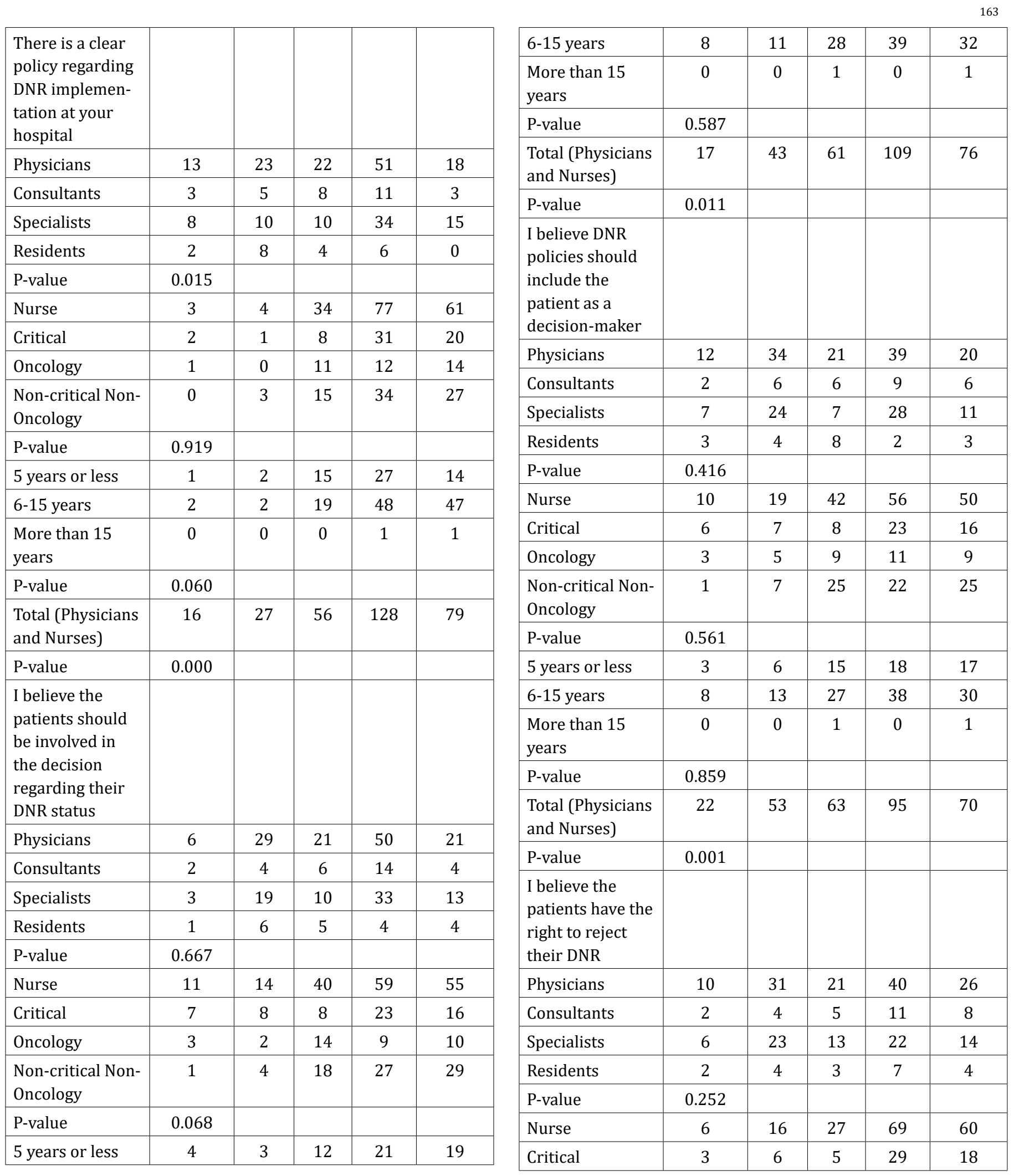




\begin{tabular}{|c|c|c|c|c|c|}
\hline Oncology & 2 & 3 & 9 & 11 & 13 \\
\hline $\begin{array}{l}\text { Non-critical Non- } \\
\text { Oncology }\end{array}$ & 1 & 7 & 13 & 29 & 29 \\
\hline P-value & 0.711 & & & & \\
\hline 5 years or less & 2 & 5 & 14 & 21 & 18 \\
\hline $6-15$ years & 5 & 10 & 13 & 48 & 40 \\
\hline $\begin{array}{l}\text { More than } 15 \\
\text { years }\end{array}$ & 0 & 0 & 1 & 0 & 1 \\
\hline P-value & 0.605 & & & & \\
\hline $\begin{array}{l}\text { Total (Physicians } \\
\text { and Nurses) }\end{array}$ & 16 & 47 & 48 & 109 & 86 \\
\hline P-value & 0.000 & & & & \\
\hline $\begin{array}{l}\text { I believe DNR de- } \\
\text { cision can result } \\
\text { in negligence of } \\
\text { patient's care }\end{array}$ & & & & & \\
\hline Physicians & 22 & 37 & 27 & 33 & 8 \\
\hline Consultants & 5 & 7 & 9 & 7 & 2 \\
\hline Specialists & 15 & 26 & 13 & 18 & 5 \\
\hline Residents & 2 & 4 & 5 & 8 & 1 \\
\hline P-value & 0.261 & & & & \\
\hline Nurse & 26 & 67 & 36 & 38 & 13 \\
\hline Critical & 9 & 28 & 4 & 15 & 6 \\
\hline Oncology & 7 & 12 & 15 & 3 & 1 \\
\hline $\begin{array}{l}\text { Non-critical Non- } \\
\text { Oncology }\end{array}$ & 10 & 27 & 17 & 20 & 6 \\
\hline P-value & 0.349 & & & & \\
\hline 5 years or less & 7 & 22 & 16 & 12 & 3 \\
\hline $6-15$ years & 20 & 43 & 18 & 27 & 10 \\
\hline $\begin{array}{l}\text { More than } 15 \\
\text { years }\end{array}$ & 0 & 1 & 1 & 0 & 0 \\
\hline P-value & 0.967 & & & & \\
\hline $\begin{array}{l}\text { Total (Physicians } \\
\text { and Nurses) }\end{array}$ & 48 & 104 & 63 & 71 & 21 \\
\hline P-value & 0.626 & & & & \\
\hline \multicolumn{6}{|l|}{$\begin{array}{l}\text { I feel comfort- } \\
\text { able discussing } \\
\text { DNR decision } \\
\text { with the patient } \\
\text { and/or the fam- } \\
\text { ily }\end{array}$} \\
\hline Physicians & 9 & 29 & 26 & 53 & 11 \\
\hline
\end{tabular}

\begin{tabular}{|c|c|c|c|c|c|}
\hline Consultants & 2 & 4 & 8 & 13 & 3 \\
\hline Specialists & 5 & 19 & 15 & 33 & 6 \\
\hline Residents & 2 & 6 & 3 & 7 & 2 \\
\hline P-value & 0.641 & & & & \\
\hline Nurse & 12 & 45 & 61 & 44 & 17 \\
\hline Critical & 1 & 15 & 18 & 20 & 7 \\
\hline Oncology & 3 & 9 & 17 & 5 & 4 \\
\hline $\begin{array}{l}\text { Non-critical Non- } \\
\text { Oncology }\end{array}$ & 8 & 21 & 26 & 19 & 6 \\
\hline P-value & 0.136 & & & & \\
\hline 5 years or less & 2 & 23 & 19 & 12 & 3 \\
\hline 6-15 years & 10 & 20 & 41 & 32 & 15 \\
\hline $\begin{array}{l}\text { More than } 15 \\
\text { years }\end{array}$ & 0 & 1 & 1 & 0 & 0 \\
\hline P-value & 0.065 & & & & \\
\hline $\begin{array}{l}\text { Total (Physicians } \\
\text { and Nurses) }\end{array}$ & 21 & 74 & 87 & 97 & 28 \\
\hline P-value & 0.145 & & & & \\
\hline
\end{tabular}

Table 4

\section{Discussion and Conclusion}

The primary aim of our study was to assess the perspectives of physicians and nurses regarding EOLC while focusing on how these perspectives were influenced by the level of education and workplace. Although there is an agreement between physicians and nurses in most of the areas we assessed, some important disagreement still exists. We further noted that differences exist between nurses based on their assigned department and level of experience.

We assessed the perspective of physicians and nurses in King Abdullah Medical City (KAMC) hospital by testing 19 statements. Both physicians and nurses agreed to 15 statements, disagreed to 2 statements, and regarding statements 4 and 7 , the nurses were having different opinions than physicians. In particular, the nurses were more likely to agree while the physicians disagree.

Nurses agreed that the patients with DNR status should always be treated in the intensive care unit if indicated. On the other hand, physicians do not want to treat DNR patients in ICU ( $\mathrm{P}=0.003)$. Similarly, nurses agreed that a religious assessment of the patients is routinely placed in their charts and nurses/physicians know about it while physicians did not agree to that $(P=0.000)$. How- 
ever, a significant difference of opinion exists among the nurses based on their duration of clinical experience and primary working departments.

Physicians and nurses both disagreed that their cultural background makes it difficult for them to deal with the DNR issue. However, their opinion is insignificant $(\mathrm{P}=0.45)$. Both nurses and physicians did not believe the DNR decision could result in negligence of patient care. This result also is not statistically important $(\mathrm{P}=$ 0.626).

Physicians and nurses of KAMC responded positively to most of the areas of assessment (15/19 statements). According to our results, one of the reasons for transferring sick patients to ICU is the fear of legal implications. Physicians and nurses both agreed that patients and families are encouraged to make timely decisions about DNR orders. Furthermore, physicians do talk about health care directives with patients and family members regularly.

No doubt religious affiliation greatly impacts the decision-making power of certain communities. As we did our study in a deeply religious society, so both physicians and nurses agreed that religious beliefs greatly influence their views about DNR. They further agreed that bereavement and follow-up services are offered to family members of patients who died in the hospital. Social workers regularly communicate with dying patients and family members in our hospital.

To know the effect of participant's position and experience on EOLC perception, our survey suggested 3 major areas of discrepancy. As compared to specialists and residents, consultants significantly agreed that effective and timely pain management is being provided to dying patients $(\mathrm{P}=0.024)$. Furthermore, consultants and specialists responded positively that there is a clear policy regarding DNR implementation in our hospital while the resident doctors disagreed $(P=0.015)$. We further noted that consultants, specialists, and residents all agreed that caring for dying patients and their families was a rewarding experience for them $(P=0.038)$.

There are certain limitations of our study. First, it is from a single-center, tertiary care hospital in the western region of Saudi Arabia, hence the generalization of its results is limited. Additionally, we did not calculate the actual practices of providing EOLC in our hospital. The possible confounding factors such as age, gender, and ethnicity have not been addressed in our cross-sectional study. Besides the above-mentioned limitations, there is a chance of having recall bias about close-ended statements as well.

\section{Publication}

The main credit in publication will go to the principal investigator and co-investigators. Those who will contribute less substantially to data collection and analysis will have an acknowledgment in the manuscript.

\section{Author Contributions}

Wrote the manuscript, searched the literature, and designed the article for submission.

\section{Conflicts of Interest}

Authors declare the absence of any conflicts of interests and their financial interest that might be construed to influence the results or interpretation of their manuscript.

\section{Acknowledgments}

Dr. Aqeel Alkhiri, Dr. Ahmad Maqadmi, Dr. Yahya Alnashri, Dr. Badr Arbaein. We would like to thank them for their contribution to the distribution and collection of questionnaire forms.

\section{Bibliography}

1. Festic E., et al. "Perspectives of Physicians and Nurses Regarding End-of-Life Care in the Intensive Care Unit". Journal of Intensive Care Medicine 27 (2012): 45-54.

2. Campbell ML and Guzman JA. "Impact of a proactive approach to improve end-of-life care in a medical ICU”. Chest 123 (2003): 266-271.

3. Yazigi A., et al. "Withholding and withdrawal of life-sustaining treatment in a Lebanese intensive care unit: a prospective observational study". Intensive Care Medicine 31 (2005): 562567.

4. Al-Jahdali HH., et al. "Advance care planning preferences among dialysis patients and factors influencing their decisions". Saudi Journal of Kidney Disease and Transplantation 20 (2009): 232-239.

5. Truog RD., et al. "Recommendations for end-of-life care in the intensive care unit: a consensus statement by the American College [corrected] of Critical Care Medicine". Critical Care Medicine 36.5 (2008): 1699. 
6. Baggs JG. "Collaborative interdisciplinary bioethical decision making in intensive care units". Nursing Outlook 41.3 (1993): 108-112.

7. Baggs JG., et al. "The dying patient in the ICU: role of the interdisciplinary team". Critical Care Clinic 20.3 (2004): 525-540.

8. Ciccarello GP. "Strategies to improve end-of-life care in the intensive care unit". Dimensions of Critical Care Nursing 22.5 (2003): 216-222.

9. Curtis JR. "Communicating about end-of-life care with patients and families in the intensive care unit". Critical Care Clinic 20.3 (2004): 363-380.

10. Al-Omari A., et al. "Critical care service in Saudi Arabia”. Saudi Medical Journal 36.6 (2015): 759-761.

11. Westphal D M., et al. "End-of- Life Decision Making in the Intensive Care Unit: Physician and Nurse Perspectives". American Journal of Medical Quality 24.3 (2009): 222-228.

12. Gouda A., et al. "Compliance with DNR policy in a tertiary care center in Saudi Arabia”. Intensive Care Medicine 36.12 (2010): 2149-2153.

13. Sha'ban Fatwas [Fatwa 12086, Part 25, pages 81-82]. The General Presidency of Scholarly Research and Ifta. Riyadh, Kingdom of Saudi Arabia (2015).

14. Aldawood AS., et al. "End-of-life practices in a tertiary intensive care unit in Saudi Arabia". Anaesthesia and Intensive Care 40.1 (2012): 137-141.

15. Al-Mobeireek A F. "Physicians' attitudes towards "do-notresuscitate" orders for the elderly: A survey in Saudi Arabia". Archives of Gerontology and Geriatrics 30.2 (2000): 151-160.

\section{Volume 5 Issue 9 September 2021}

(C) All rights are reserved by Naved Yousuf Hasan., et al. 